P. Orlik and L. Solomon

Nagoya Math. J.

Vol. 109 (1988), 1-21

\title{
THE HESSIAN MAP IN THE INVARIANT THEORY OF REFLECTION GROUPS
}

\author{
PETER ORLIK AND LOUIS SOLOMON*)
}

\section{§1. Introduction}

Let $V$ be a complex vector space of dimension $l$. Let $S$ be the $C$-algebra of polynomial functions on $V$. Let $\operatorname{Der}_{S}$ be the $S$-module of derivations of $S$ and let $\Omega_{S}=\operatorname{Hom}_{S}\left(\operatorname{Der}_{S}, S\right)$ be the dual $S$-module of differential 1-forms. Let $\left\{e_{i}\right\}$ be a basis for $V$ and let $\left\{x_{i}\right\}$ be the dual basis for $V^{*}$. Then $\left\{D_{i}=\partial / \partial x_{i}\right\}$ and $\left\{d x_{i}\right\}$ are bases for $\operatorname{Der}_{S}$ and $\Omega_{S}$ as $S$-modules. If $f \in S$, define a map Hess $(f): \operatorname{Der}_{S} \rightarrow \Omega_{S}$ by

$$
\operatorname{Hess}(f): \theta \longrightarrow \sum \theta\left(D_{i} f\right) d x_{i} \quad \theta \in \operatorname{Der}_{s} .
$$

Then Hess $(f)$ is an $S$-module homomorphism which does not depend on the choice of basis for $V$. Let $\mathbf{H}(f)$ denote the the matrix of the map Hess $(f)$ with respect to the pair of bases $\left\{D_{i}\right\}$ and $\left\{d x_{i}\right\}$. Then $\mathbf{H}(f)$ is the usual Hessian matrix of second partial derivatives of $f$.

Let $G \subset G L(V)$ be a finite unitary reflection group and let $R=S^{G}$ be the subalgebra of $G$-invariant polynomials. Both $\operatorname{Der}_{S}$ and $\Omega_{S}$ are $G$ modules. If $f \in R$ then Hess $(f)$ induces a homomorphism again denoted Hess $(f): \operatorname{Der}_{S}^{G} \rightarrow \Omega_{S}^{G}$. If $G$ has a real form, so that $G$ is a Coxeter group, then it has a non-degenerate invariant quadratic form $f$. Nondegeneracy implies $\operatorname{det} \mathbf{H}(f) \neq 0$. Since $\mathbf{H}(f)$ is a matrix with entries in $C$ it is invertible in $M_{l}(C)$ so Hess $(f): \operatorname{Der}_{S}^{G} \rightarrow \Omega_{S}^{G}$ is an isomorphism [15]. The situation is more complicated for unitary reflection groups which do not have a real form. We show that if Hess $(f)$ is an isomorphism then $f$ is an invariant form of minimal positive degree but this minimality condition is not sufficient. In Theorem (5.10) of this paper we characterize those irreducible unitary reflection groups for which an invariant form $f_{1}$ of minimal positive degree induces an isomorphism $\operatorname{Hess}\left(f_{1}\right): \operatorname{Der}_{S}^{G} \rightarrow \Omega_{S}^{G}$.

Received February 6, 1986.

*) This work was supported in part by the National Science Foundation. 
Shephard $[17,18]$ introduced the notion of a regular complex polytope $\mathscr{P}$ and showed that its symmetry group $G=\operatorname{Aut}(\mathscr{P})$ is a finite irreducible unitary reflection group. A regular convex polytope in $R^{l}$ defines a regular complex polytope in $V=C^{l}$ by scalar extension; if $\mathscr{P}$ arises in this way we say that $\mathscr{P}$ has a real form. If $\mathscr{P}$ has a real form then $G$ is a finite Coxeter group.

(1.2) Definition. A Shephard group is the symmetry group of a regular complex polytope.

Shephard groups have been classified and studied in the work of Shephard [17, 18], Shephard and Todd [19], Coxeter [2, 3, 4] and Koster [10]. There are irreducible unitary reflection groups which are not Shephard groups. In Table 1 we list the Shephard groups which are not Coxeter groups and some relevant information about each group. If $l \geq 3$ our arguments depend in part on this classification. Our main result is:

(1.3) Theorem. Let $G \subset G L(V)$ be a Shephard group and let $f_{1} \in R$ be a G-invariant form of minimal positive degree. Then the map Hess $\left(f_{1}\right)$ : $\operatorname{Der}_{S}^{G} \rightarrow \Omega_{S}^{G}$ is an isomorphism.

By Chevalley's theorem there exist forms $f_{1}, \cdots, f_{l} \in R$ called basic invariants such that $R=C\left[f_{1}, \cdots, f_{l}\right]$. Let $d_{i}=\operatorname{deg} f_{i}$ and number the $f_{i}$ so that $d_{1} \leq \cdots \leq d_{l}$. Let $m_{i}=d_{i}-1$ be the exponents of $G$. The $R$ module $\Omega_{S}^{G}$ is free of rank $l$ with basis $d f_{1}, \cdots, d f_{l}$. The $R$-module Der Der $_{S}^{G}$ is free of rank $l$ with a basis of homogeneous elements $\theta_{1}, \cdots, \theta_{l}$ called basic derivations. Let $K$ be the quotient field of $R$. Since $K \otimes \operatorname{Der}_{S}^{G}$ and $K \otimes$ $\Omega_{S}^{G}$ are vector spaces over $K$ of the same finite dimension $l$, it suffices in (1.5) to prove that Hess $\left(f_{1}\right)$ is surjective. However, our argument uses injectivity in the proof of surjectivity. To prove that Hess $\left(f_{1}\right)$ is injective it suffices to show that $\operatorname{det} \mathbf{H}\left(f_{1}\right) \neq 0$. The vanishing of the Hessian determinant has an interesting history which we outline briefly in Section 4. To prove that Hess $\left(f_{1}\right)$ is surjective it suffices, since the $d f_{i}$ generate $\Omega_{S}^{G}$ as $R$-module, to prove that $d f_{i} \in i m \operatorname{Hess}\left(f_{1}\right)$. Let $\theta_{E}=\sum x_{i} D_{i}$ be the Euler derivation. Since $\theta_{E}\left(D_{i} f_{1}\right)=m_{1}\left(D_{i} f_{1}\right)$ it follows from (1.1) that Hess $\left(f_{1}\right) \theta_{E}=m_{1} d f_{1}$. We prove that for given basic invariants $f_{1}, \cdots, f_{l}$ there exist basic derivations $\theta_{1}, \cdots, \theta_{l}$ such that

$$
\operatorname{Hess}\left(f_{1}\right) \theta_{i}=m_{1} d f_{i} \quad 1 \leq i \leq l .
$$

This forces $\theta_{1}=\theta_{E}$. The assertion (1.4) and hence (1.3) is equivalent to 
a statement about matrices over $S$. To give the matrix formulation we make the following definitions. If $f_{1}, \cdots, f_{l} \in S$ define the Jacobian matrix $\mathbf{J}=\mathbf{J}\left(f_{1}, \cdots, f_{l}\right)$ by $\mathbf{J}_{i j}=D_{i} f_{j}$. If $\theta_{1}, \cdots, \theta_{l} \in \operatorname{Der}_{s}$ define a matrix $\mathbf{Q}=$ $\mathbf{Q}\left(\theta_{1}, \cdots, \theta_{l}\right)$ by $\mathbf{Q}_{i j}=\theta_{j}\left(x_{i}\right)$. Thus $\theta_{j}=\sum \mathbf{Q}_{i j} D_{i}$. The matrices $\mathbf{J}\left(f_{1}, \cdots\right.$, $\left.f_{l}\right)$ and $\mathbf{Q}\left(\theta_{1}, \cdots, \theta_{l}\right)$ depend on the chosen basis $\left\{e_{i}\right\}$. It will be convenient to use the notation $J\left(f_{1}, \cdots, f_{l}\right)=\operatorname{det} \mathbf{J}\left(f_{1}, \cdots, f_{l}\right), Q\left(\theta_{1}, \cdots, \theta_{l}\right)=\operatorname{det} \mathbf{Q}\left(\theta_{1}\right.$, $\left.\cdots, \theta_{l}\right)$ and $H(f)=\operatorname{det} \mathbf{H}(f)$.

(1.5) Theorem. Let $G \subset G L(V)$ be a Shephard group and let $f_{1}, \cdots, f_{l}$ be a set of basic invariants, with $f_{1}$ of minimal positive degree. Then there exist basic derivations $\theta_{1}, \cdots, \theta_{l}$ such that

$$
\mathbf{H}\left(f_{1}\right) \mathbf{Q}\left(\theta_{1}, \cdots, \theta_{l}\right)=m_{1} \mathbf{J}\left(f_{1}, \cdots, f_{l}\right) .
$$

The equivalence of (1.3) and (1.5) is clear via (1.4). Note that the formula (1.5) contains the assertion $H\left(f_{1}\right) \neq 0$ because $J\left(f_{1}, \cdots, f_{l}\right) \neq 0$ by the algebraic independence of $f_{1}, \cdots, f_{l}$.

If $l=2$ then our argument in Section 3 yields formulae relating Klein's vertex, face and edge forms of the regular polyhedra which we believe to be new. Let $\mathscr{P}$ be one of the three polyhedra: tetrahedron, octahedron, icosahedron. Let $\varphi_{V}=\varphi_{V}(\mathscr{P}), \varphi_{F}=\varphi_{F}(\mathscr{P})$, and $\varphi_{E}=\varphi_{E}(\mathscr{P})$ be the forms which Klein [9, Chapter 2] has associated with the vertices, faces, and edges of $\mathscr{P}$ :

\begin{tabular}{cccc}
$\mathscr{P}$ & $\varphi_{V}$ & $\varphi_{F}$ & $\varphi_{E}$ \\
\hline tetrahedron & $\Phi$ & $\Psi$ & $t$ \\
octahedron & $t$ & $W$ & $\chi$ \\
icosahedron & $f$ & $H$ & $T$
\end{tabular}

Klein showed that

$$
\begin{aligned}
\varphi_{F} & \approx H\left(\varphi_{V}\right) \\
\varphi_{E} & \approx J\left(\varphi_{V}, \varphi_{F}\right) .
\end{aligned}
$$

Here and throughout this paper the symbol $\approx$ means that the forms are equal up to a nonzero constant multiple. It follows from our results in Section 3 applied to certain Shephard groups in two dimensions that with $q=3,4,5$ for the tetrahedron, octahedron, icosahedron we have

$$
\begin{aligned}
& \varphi_{F}^{2} \approx J\left(\varphi_{V}, \varphi_{E}\right) \\
& \varphi_{V}^{q-1} \approx J\left(\varphi_{F}, \varphi_{E}\right)
\end{aligned}
$$




$$
D_{i} \varphi_{E} \approx J\left(D_{i} \varphi_{v}, \varphi_{F}\right) \quad i=1,2
$$

for each of the three polyhedra $\mathscr{P}$. The constant implied by $\approx$ in (1.10) is the same for $i=1,2$. Note that (1.7) follows from (1.10) and the Euler formula but that (1.10) is not a formal consequence of (1.7). Klein [9, pps. 62-63] also remarks that "apart from trivial exceptions" the vertex forms $\varphi_{V}$ of the three polyhedra are characterized among all binary forms by the vanishing of their fourth transvectant. At the end of Section 3 we discuss the connection between this vanishing property and our work in this paper.

In Section 2 we prove some general lemmas about the matrices $\mathbf{Q}\left(\theta_{1}\right.$, $\left.\cdots, \theta_{l}\right), \mathbf{J}\left(f_{1}, \cdots, f_{l}\right)$ and $\mathbf{H}(f)$. In Section 3 we prove (1.3) for $l=2$. In Section 4 we prove (1.3) for $l \geq 3$. Our argument in case $l=2$ does not use the classification. In case $l \geq 3$ it does. In Section 5 we deduce some consequences of (1.3). In particular there is an inclusion of ideals

$$
\left(f_{1}, \cdots, f_{l}\right) \subseteq\left(D_{1} f_{1}, \cdots, D_{l} f_{1}\right) .
$$

This shows that if $G$ is a Shephard group then an invariant form $f_{1}$ of minimal positive degree is nondegenerate. In the sequel [14] we use (1.5) to prove that if $G$ is a Shephard group, then the complement of the union of its reflecting hyperplanes is a $K(\pi, 1)$ space. In [14] we also give, for all Shephard groups, explicit basic derivations $\theta_{1}, \cdots, \theta_{l}$ and basic invariants $f_{1}, \cdots, f_{l}$ which satisfy the matrix equation of (1.5).

We would like to thank Hiroaki Terao for many interesting discussions on arrangements of hyperplanes, which convinced us that our ideas on $S \otimes V$ are more naturally stated in terms of $\operatorname{Der}_{S}$.

\section{§2. General lemmas}

In this section $G$ is any finite irreducible unitary reflection group. In fact it would suffice in (2.1)-(2.18) to assume that $G$ is any subgroup of $G L(V)$. We use the notation of Section 1. Let $\langle x, v\rangle$ denote the natural pairing $V^{*} \times V \rightarrow C$ and let $\langle\omega, \theta\rangle$ denote the natural pairing $\Omega_{S} \times \operatorname{Der}_{S}$ $\rightarrow S$. If $v \in V$ let $D_{v} \in \operatorname{Der}_{S}$ be the derivation defined by $D_{v} x=\langle x, v\rangle$ for $x \in V^{*}$. If $f \in S$ then $d f \in \Omega_{S}$ is defined by $\langle d f, \theta\rangle=\theta(f)$ for $\theta \in \operatorname{Der}_{S}$. In terms of the bases $\left\{D_{i}=\partial / \partial x_{i}\right\}$ and $\left\{d x_{i}\right\}$ we have $d f=\sum\left(D_{i} f\right) d x_{i}$ and $\theta(f)=\sum \theta\left(x_{i}\right) D_{i} f$

The spaces $S, \operatorname{Der}_{S}$, and $\Omega_{S}$ have $G$-module structures. We define the $G$-actions and list some transformation formulas. Let $g \in G, v \in V, a \in S$, 
$\theta \in \operatorname{Der}_{S}$, and $\omega \in \Omega_{S}$. The $G$-module structure in $S$ is defined by

$$
(g a)(v)=a\left(g^{-1} v\right) .
$$

It follows that

$$
\begin{gathered}
g\left(D_{v} a\right)=D_{g v}(g a) \\
d(g a)=g(d a) .
\end{gathered}
$$

The $G$-module structure in $\operatorname{Der}_{S}$ is defined by

$$
(g \theta)(a)=g\left(\theta\left(g^{-1} a\right)\right) .
$$

It follows that

$$
\begin{gathered}
g D_{v}=D_{g v} \\
g(a \theta)=(g a)(g \theta) .
\end{gathered}
$$

The $G$-module structure in $\Omega_{S}$ is defined by

$$
(g \omega)(\theta)=g\left(\omega\left(g^{-1} \theta\right)\right) .
$$

It follows that

$$
\begin{gathered}
g(a \omega)=(g a)(g \omega) \\
\langle g \omega, g \theta\rangle=g\langle\omega, \theta\rangle .
\end{gathered}
$$

We give $M_{l}(S)$ the $G$-module structure

$$
(g \mathbf{P})_{i j}=g\left(\mathbf{P}_{i j}\right) \quad \mathbf{P} \in M_{l}(S) .
$$

If $g \in G$ let $[g]$ denote the matrix for $g$ in the basis $\left\{e_{i}\right\}$ so that $[g]_{i j}=$ $\left\langle x_{j}, g e_{i}\right\rangle$. The following transformation rules are easy consequences of (2.1)-(2.10).

(2.11) If $f \in R$ then $g \mathbf{H}(f)=[g]^{T} \mathbf{H}(f)[g]$.

(2.12) If $f_{1}, \cdots, f_{l} \in R$ and $\mathbf{J}=\mathbf{J}\left(f_{1}, \cdots, f_{l}\right)$ then $g \mathbf{J}=[g]^{T} \mathbf{J}$.

(2.13) If $\theta_{1}, \cdots, \theta_{l} \in \operatorname{Der}_{S}^{G}$ and $\mathbf{Q}=\mathbf{Q}\left(\theta_{1}, \cdots, \theta_{l}\right)$ then $g \mathbf{Q}=\left[g^{-1}\right] \mathbf{Q}$.

(2.14) Lemma. If $f \in R$ then Hess $(f): \operatorname{Der}_{s} \rightarrow \Omega_{S}$ is a G-module homomorphism and thus induces a map $\operatorname{Hess}(f): \operatorname{Der}_{S}^{f} \rightarrow \Omega_{S}^{G}$.

Proof. Let $g \in G$. First suppose $f \in S$ is any polynomial. Let $h=$ Hess $(f)$. From (1.1) we get 


$$
h D_{v}=\sum D_{v}\left(D_{i} f\right) d x_{i}=\sum D_{i}\left(D_{v} f\right) d x_{i}=d\left(D_{v} f\right) .
$$

It follows from (2.15) and (2.1)-(2.8) that $g\left(h D_{v}\right)=g\left(d\left(D_{v} f\right)\right)=d\left(g\left(D_{v} f\right)\right)$ $=d\left(D_{g v} g f\right)$ and $h\left(g D_{v}\right)=h\left(D_{g v}\right)=d\left(D_{g v} f\right)$ for all $v \in V$. Thus if $f \in R$ then $g\left(h D_{v}\right)=h\left(g D_{v}\right)$. Let $a \in S$. Since $h$ is an $S$-module map we have $g\left(h\left(a D_{v}\right)\right)=g\left(a\left(h D_{v}\right)\right)=(g a)\left(g\left(h D_{v}\right)\right)=(g a)\left(h\left(g D_{v}\right)\right)=h\left((g a)\left(g D_{v}\right)\right)=$ $h\left(g\left(a D_{v}\right)\right)$. The result follows because $\operatorname{Der}_{S}=\sum S D_{v}$.

The $S$-modules $\operatorname{Der}_{S}$ and $\Omega_{S}$ are graded as follows. Give $S=\bigoplus_{p \geq 0} S_{p}$ its usual grading so that $S_{1}=V^{*}$. We call nonzero elements of $S_{p}$ forms of degree $p$. Grade $\operatorname{Der}_{S}$ by

$$
\theta \in\left(\operatorname{Der}_{S}\right)_{q} \text { if } \theta S_{p} \subseteq S_{p+q} \text { for all } p \geq 0
$$

and grade $\Omega_{S}$ by

$$
\omega \in\left(\Omega_{S}\right)_{q} \text { if } \omega\left(\left(\operatorname{Der}_{S}\right)_{p}\right) \subseteq S_{p+q} \text { for all } p \geq 0 .
$$

Thus $D_{i}$ has degree -1 and $d x_{i}$ has degree +1 . It follows from (2.14) and this grading that:

(2.18) Lemma. If $f \in R$ is a form of degree $p$ then $\operatorname{Hess}(f): \operatorname{Der}_{S}^{G} \rightarrow$ $\Omega_{S}^{G}$ is a graded $R$-module map of degree $p$.

The main result of this paper concerns an invariant form of minimal positive degree. The significance of minimality is made clear by the following lemma.

(2.19) Lemma. Let $f$ be an invariant form. If $\operatorname{Hess}(f): \operatorname{Der}_{S}^{G} \rightarrow \Omega_{S}^{G}$ is an epimorphism then $f$ is an invariant form of minimal positive degree.

Proof. Suppose $f \in R_{p}$ and suppose that $R_{q} \neq 0$ for some positive integer $q$. Then $\left(\Omega_{S}^{G}\right)_{q} \supseteq d R_{q} \neq 0$. Since Hess $(f)$ is a surjective map of degree $p$ we have $\left(\operatorname{Der}_{S}^{G}\right)_{q-p} \neq 0$. Since $G$ is an irreducible group we have $\left(\operatorname{Der}_{S}^{G}\right)_{-1}$ $=0$. Thus $q-p \geq 0$.

Each reflection in $G$ fixes a hyperplane in $V$. Let $\mathscr{A}=\mathscr{A}(G)$ be the set of these hyperplanes and let $n=|\mathscr{A}|$. If $H \in \mathscr{A}$ let $\alpha_{H} \in V^{*}$ be a linear form with kernel $H$. The subgroup of $G$ fixing $H$ is cyclic. Let $s_{H}$ be a generator of this cyclic group and let $e_{H}$ be its order. Let $m$ be the number of reflections in $G$. Then

$$
m=\sum_{H \in \mathscr{A}}\left(e_{H}-1\right), \quad n=\sum_{H \in \mathscr{A}} 1 .
$$


(2.21) Lemma. Let $f_{1}, \cdots, f_{l}$ be basic invariants for $G$. The R-module $\Omega_{S}^{G}$ is free of rank $l$ with basis $d f_{1}, \cdots, d f_{l}$. The $R$-module $\operatorname{Der}_{S}^{F}$ is free of rank $l$ with a basis of homogeneous elements.

Proof. If $M$ is a vector space over $C$ we give $S \otimes M$ the $S$-module structure $a(b \otimes m)=a b \otimes m$, the $G$-module structure $g(a \otimes m)=g a \otimes g m$ and the grading $(S \otimes M)_{p}=S_{p} \otimes M$. Define $S$-module isomorphisms $\alpha$ : $S \otimes V \rightarrow \operatorname{Der}_{S}$ and $\beta: S \otimes V^{*} \rightarrow \Omega_{S}$ by $\alpha(a \otimes v)=a D_{v}$ and $\beta(a \otimes x)=a d x$. It follows from (2.1)-(2.8) that both $\alpha$ and $\beta$ are $G$-module homomorphisms and thus, by restriction, define $R$-module isomorphisms

$$
\alpha:(S \otimes V)^{G} \longrightarrow \operatorname{Der}_{S}^{G} \text { and } \beta:\left(S \otimes V^{*}\right)^{G} \longrightarrow \Omega_{S}^{G} .
$$

These maps are homogeneous with degrees: $\operatorname{deg} \alpha=-1$ and $\operatorname{deg} \beta=+1$. It is shown in [20, Lemma $2 ; 13,(2.3)]$ that if $M$ is any $G$-module of finite dimension, then $(S \otimes M)^{G}$ is a free $R$-module of rank equal to $\operatorname{dim}_{C} M$. Apply this with $M=V$ and $M=V^{*}$. The assertions of the lemma follow from the $R$-module isomorphisms (2.22).

We call a homogeneous $R$-basis for $\operatorname{Der}_{S}^{f}$ a set of basic derivations. Let $\left\{\theta_{1}, \cdots, \theta_{l}\right\}$ be a set of basic derivations. Define integers $n_{i}$ by $\operatorname{deg} \theta_{i}$ $=n_{i}-1$. Our definition of the integers $n_{i}$ here agrees with the definition of the coexponents $n_{i}$ in [13] because the isomorphism $\alpha$ in (2.22) has degree -1 . It follows from [13, (2.3) ff.] that the integers $n_{i}$ do not depend on the choice of basic derivations. We agree to number the $n_{i}$ so that $n_{1} \leq n_{2} \leq \cdots \leq n_{l}$.

(2.23) Lemma. (i) $1=n_{1}<n_{2}$. (ii) If $\theta_{E}=\sum x_{i} D_{i}$ is the Euler derivation then $\theta_{1}=\theta_{E}$.

Proof. Since $G$ is an irreducible group we have $\left(\operatorname{Der}_{S}^{G}\right)_{-1}=0$. Thus it suffices to show that $\left(\operatorname{Der}_{S}^{G}\right)_{0}=C \theta_{E}$. Let $\alpha$ be the isomorphism in (2.22). Then $\left(\operatorname{Der}_{S}^{G}\right)_{0}=\alpha\left(\left(S_{1} \otimes V\right)^{G}\right)=\alpha\left(\left(V^{*} \otimes V\right)^{G}\right)$. Since $G$ is irreducible we have $\left(V^{*} \otimes V\right)=C \sum x_{i} \otimes e_{i}$. The assertion follows since $\alpha\left(\sum x_{i} \otimes e_{i}\right)=\theta_{E}$.

Define polynomials $J$ and $Q$ by

$$
J=\prod_{H \in \mathscr{A}} \alpha_{H}^{e_{H}-1} \quad Q=\prod_{H \in \mathscr{A}} \alpha_{H} .
$$

It follows from (2.20) that $\operatorname{deg} J=m$ and $\operatorname{deg} Q=n$. It is known [19, $(8.3) ; 13,(3.11)]$ that

$$
\sum m_{i}=m \text { and } \sum n_{i}=n
$$


If $g \in G$ let $\delta(g)=\operatorname{det} g$. Recall the notation

$$
J\left(f_{1}, \cdots, f_{l}\right)=\operatorname{det} \mathbf{J}\left(f_{1}, \cdots, f_{l}\right) \text { and } Q\left(\theta_{1}, \cdots, \theta_{l}\right)=\operatorname{det} \mathbf{Q}\left(\theta_{1}, \cdots, \theta_{l}\right) .
$$

(2.26) Lemma. Let $f_{1}, \cdots, f_{l}$ be basic invariants. Then (i) $g J\left(f_{1}, \cdots\right.$, $\left.f_{l}\right)=\delta(g) J\left(f_{1}, \cdots, f_{l}\right)$, (ii) $J\left(f_{1}, \cdots, f_{l}\right) \approx J$, (iii) If $a \in S$ and $g a=\delta(g) a$ for all $g \in G$ then $a \in R J$.

Proof. These facts are known: (i) follows from (2.12); (ii) is proved in $[19,(5.2)]$; (iii) is given in $[1,5.5$, Prop. 6].

(2.27) Lemma. Let $\theta_{1}, \cdots, \theta_{l}$ be basic derivations. Then (i) $g Q\left(\theta_{1}, \cdots\right.$, $\left.\theta_{l}\right)=\delta\left(g^{-1}\right) Q\left(\theta_{1}, \cdots, \theta_{l}\right)$, (ii) $Q\left(\theta_{1}, \cdots, \theta_{l}\right) \approx Q$, (iii) If $a \in S$ and $g a=\delta\left(g^{-1}\right) a$ for all $g \in G$ then $a \in R Q$.

Proof. (i) follows from (2.13). We prove (ii) and (iii) simultaneously. Suppose $a \in S$ and $g a=\delta\left(g^{-1}\right) a$ for all $g \in G$. Let $H \in \mathscr{A}$ and let $v \in H$. Then $a(v)=a\left(s_{H} v\right)=\left(s_{H}^{-1} a\right)(v)=\delta\left(s_{H}\right) a(v)$. Since $\delta\left(s_{H}\right) \neq 1$ we have $a(v)=0$. Thus $\alpha_{H}$ divides $a$. This is true for all $H \in \mathscr{A}$ so $Q$ divides $a$. In particular with $a=Q\left(\theta_{1}, \cdots, \theta_{l}\right)$ it follows from (i) that $Q$ divides $Q\left(\theta_{1}, \cdots, \theta_{l}\right)$. We know from [13, (2.5)] applied to $M=V^{*}$, and the isomorphism $\alpha$ of (2.22), that $Q\left(\theta_{1}, \cdots, \theta_{l}\right) \neq 0$. From (2.24) and (2.25) we have $\operatorname{deg} Q\left(\theta_{1}, \cdots\right.$, $\left.\theta_{l}\right)=\sum n_{i}=n=\operatorname{deg} Q$. This proves (ii) and hence (iii).

Note that although the matrices $\mathbf{J}\left(f_{1}, \cdots, f_{l}\right)$ and $\mathbf{Q}\left(\theta_{1}, \cdots, \theta_{l}\right)$ depend on choice of $f_{1}, \cdots, f_{l}$ and $\theta_{1}, \cdots, \theta_{l}$ their determinants $J\left(f_{1}, \cdots, f_{l}\right) \approx J$ and $Q\left(\theta_{1}, \cdots, \theta_{l}\right) \approx Q$ are nonzero polynomials uniquely determined up to a constant multiple.

The next lemma is an analog of a result of Saito [16, Theorem 1.8. ii].

(2.28) Lemma. A set $\eta_{1}, \cdots, \eta_{l}$ of homogeneous elements of $\operatorname{Der}_{S}^{G}$ is a set of basic derivations if and only if $Q\left(\eta_{1}, \cdots, \eta_{l}\right) \approx Q$.

Proof. If $\eta_{1}, \cdots, \eta_{l}$ is a set of basic derivations then (2.27. ii) shows that $Q\left(\eta_{1}, \cdots, \eta_{l}\right) \approx Q$. Conversely suppose $Q\left(\eta_{1}, \cdots, \eta_{l}\right) \approx Q$. Let $\theta_{1}, \cdots, \theta_{l}$ be a set of basic derivations. Write $\eta_{i}=\sum b_{j i} \theta_{j}$ and let $B=\left[b_{i j}\right]$. Then $Q\left(\eta_{1}, \cdots, \eta_{l}\right)=\operatorname{det}(B) Q\left(\theta_{1}, \cdots, \theta_{2}\right)$. The hypothesis and (2.27. ii) imply $\operatorname{det}(B) \in C^{*}$ so the conclusion follows.

(2.29) Lemma. If $f \in R$ then (i) $g H(f)=\delta(g)^{2} H(f)$ and (ii) $H(f) Q \in R J$.

Proof. Assertion (i) follows from (2.11). It follows from (i) and (2.27. i, ii) that $g(H(f) Q)=\delta(g) H(f) Q$. Now (2.26. iii) shows that $H(f) Q \in R J$. 
(2.30) Lemma. Let $f \in R_{p}$ and suppose $H(f) \neq 0$. Then (i) $l(p-2)=$ $m-n+d \geq m-n$ where $d$ is the degree of an invariant form; (ii) If $l(p-2)=m-n$ then $H(f) \approx J / Q$.

Proof. Since $H(f) \neq 0$ it follows from (2.29. ii) that $H(f)=a J / Q$ for some form $a \in R$ of degree say $d$. Then (i) follows by comparing degrees. If $l(p-2)=m-n$ then $d=0$ so $a$ is constant. This proves (ii).

\section{§3. The two-dimensional case}

In this section we assume that $\operatorname{dim} V=2$.

(3.1) Lemma. Let $f \in S$ be a form. If $H(f)=0$ then $f$ is a power of a linear form.

Proof. This is part of 19th century invariant theory [6, p. 235]. Since there is an easy argument [21, Ex. 3.3.14] we give it here. By the Euler formula we have $\left|\begin{array}{ll}D_{11} f & D_{12} f \\ D_{1} f & D_{2} f\end{array}\right|=0$. Let $x$ be an indeterminate and define a homomorphism $\pi: S \rightarrow C[x]$ by $\pi\left(x_{1}\right)=x$ and $\pi\left(x_{2}\right)=1$. Note that no form lies in the kernel of $\pi$. Let $D=d / d x$. Then $\pi\left(D_{1} u\right)=D(\pi u)$ for all $u \in S$. Let $u_{i}=\pi\left(D_{i} f\right)$ for $i=1,2$. Then $\left|\begin{array}{cc}D u_{1} & D u_{2} \\ u_{1} & u_{2}\end{array}\right|=0$. If $u_{1}=0$ then $f \in C x_{2}^{d}$ where $d=\operatorname{deg} f$ and the assertion is clear. If $u_{1} \neq 0$ then $D\left(u_{2} / u_{1}\right)$ $=0$ so $u_{2} / u_{1} \in C$. Say $u_{2}=c u_{1}$. Then $\pi\left(D_{2}-c D_{1}\right) f=0$ so $\left(D_{2}-c D_{1}\right) f=0$ and the assertion follows.

(3.2) Lemma. If $G \subset G L(V)$ is an irreducible group and $f \in S$ is a $G$-invariant form of positive degree then $H(f) \neq 0$.

Proof. This follows from (3.1).

(3.3) Lemma. Let $G \subset G L(V)$ be an irreducible unitary reflection group. Then $2\left(d_{1}-2\right)=m-n+d$ where $d \in\left\{0, d_{1}, d_{2}\right\}$.

Proof. Lemma (3.2) shows $H(f) \neq 0$ so we may apply (2.30) to conclude that $2\left(d_{1}-2\right)=m-n+d$ where $d$ is the degree of an invariant form. Since $R=C\left[f_{1}, f_{2}\right]$ we may write $d=a_{1} d_{1}+a_{2} d_{2}$ where $a_{1}, a_{2}$ are non-negative integers. Since $m \geq n$ we have $\left(a_{1}-2\right) d_{1}+a_{2} d_{2} \leq-4$. Thus $a_{1}=0,1$. If $a_{1}=0$ then $a_{2}=0,1$ because $d_{1} \leq d_{2}$. If $a_{1}=1$ then $a_{2}=0$ because $d_{1} \leq d_{2}$.

(3.4) Remark. All three possibilities $d=0, d_{1}, d_{2}$ do in fact occur. 
By (2.25) we have $m=d_{1}+d_{2}-2$ so the formula in the lemma is equivalent to $m+n=2 d_{2}+d$ where $d \in\left\{0, d_{1}, d_{2}\right\}$. We observed in [13, Theorem 5.4] that $m+n \geq l d_{l}$ for all irreducible reflection groups. Thus Lemma (3.3) gives a case free argument for this inequality if $l=2$.

(3.5) Theorem. If $G \subset G L(V)$ is a Shephard group then $2\left(d_{1}-2\right)=$ $m-n$ and thus $m+n=2 d_{2}$.

Proof. If we can show that

$$
n<d_{2}+2
$$

then, in the notation of (3.3), we have $d=n+d_{1}-d_{2}-2<d_{1}$, so $d=0$ by minimality of $d_{1}$ and the theorem is proved. Since $G$ is a Shephard group, it follows from work of Coxeter [4, pps. 94-5] that $G$ may be generated by two reflections $s_{1}, s_{2}$ and there exist positive integers $p_{1}, p_{2}, q$ such that

$$
\begin{aligned}
& s_{1}^{p_{1}}=1 \quad s_{2}^{p_{2}}=1 \\
& s_{1} s_{2} s_{1} \cdots=s_{2} s_{1} s_{2} \cdots
\end{aligned}
$$

where there are $q$ terms on both sides of the last equation. Let $H_{i} \in \mathscr{A}$ be the hyperplane fixed by $s_{i}$ and let $\mathcal{O}_{i} \subseteq \mathscr{A}$ be the $G$-orbit of $H_{i}$ in the natural action of $G$ on $\mathscr{A}$. Let $\mathcal{O}$ be any orbit of $G$ on $\mathscr{A}$. If $\mathcal{O} \notin\left\{\mathcal{O}_{1}, \mathcal{O}_{2}\right\}$ it follows from [21, Theorem 4.3.4. i] that there exists a homomorphism $\lambda: G \rightarrow C^{*}$ such that $\lambda\left(s_{i}\right)=1$ for $i=1,2$ and $\lambda(s) \neq 1$ for any reflection $s$ which fixes a hyperplane $H \in \mathcal{O}$. This contradicts the fact that $s_{1}, s_{2}$ generate $G$. Thus $\mathcal{O} \in\left\{\mathcal{O}_{1}, \mathcal{O}_{2}\right\}$ so $\mathscr{A}=\mathcal{O}_{1} \cup \mathcal{O}_{2}$.

Let $E_{i}$ be the cyclic subgroup fixing $H_{i}$ and let $e_{i}=\left|E_{i}\right|$. Then $e_{i} \geq p_{i}$. (In fact equality holds but we do not need this.) Suppose $\mathcal{O}_{1}=\mathcal{O}_{2}$. Then $m=\left(e_{1}-1\right)\left|\mathcal{O}_{1}\right|=\left(e_{1}-1\right) n$. If $e_{1}=2$ then $p_{1}=2=p_{2}$ so $G$ is a dihedral group. In this case $d_{1}=2$ and $m=n$ so (3.5) is clear. If $e_{1} \geq 3$ then $m \geq 2 n$ so $d_{1}+d_{2}-2 \geq 2 n$ and (3.6) is clear. Thus we may assume $\mathcal{O}_{1} \neq \mathcal{O}_{2}$. If $q$ is odd then $s_{1} s_{2} s_{3} \ldots=s_{1} s_{2} s_{3} \ldots$ shows that $s_{1}$ and $s_{2}$ are conjugate in $G$ so $\mathcal{O}_{1}=\mathcal{O}_{2}$, a contradiction. Thus $q$ is even. Let $F_{i}=\left\{g \in G \mid g H_{i} \subseteq H_{i}\right\}$ and let $Z$ be the center of $G$. Since $Z E_{i} \subseteq F_{i}$ and $Z \cap E_{i}=1$ we have $\left|F_{i}\right| \geq|Z| e_{i}$. Thus

$$
|G: Z| \geq e_{i}\left|G: F_{i}\right|=e_{i}\left|\mathcal{O}_{i}\right| \quad(i=1,2) .
$$

Coxeter [4, p. 154] has shown that 


$$
d_{1}=2 h / q, \quad d_{2}=h
$$

where $h$ is the order of $s_{1} s_{2}$. Since $q$ is even it follows that $d_{1}$ divides $d_{2}$. It is known [22, (3.3)] that $|Z|=$ g.c.d. $\left(d_{1}, d_{2}\right)$. In our case this says $|Z|$ $=d_{1}$. By $[19,(5.1)]$ we have $|G|=d_{1} d_{2}$ and thus $|G: Z|=d_{2}$. It follows from (3.7) that

$$
2 d_{2} \geq e_{1}\left|\mathcal{O}_{1}\right|+e_{2}\left|\mathcal{O}_{2}\right|=m+n=d_{1}+d_{2}-2+n
$$

which proves (3.6).

We prove a general lemma about binary forms which helps us to prove (1.5). Let $f, \varphi$ be binary forms of degrees $d, n$. If $r$ is a non-negative integer, the $r$-th transvectant $(f, \varphi)^{r}$ of $f$ and $\varphi$ is defined [6, p. 46] by

$$
(f, \varphi)^{r}=\frac{(d-r) !(n-r) !}{d !} n ! \sum_{k=0}^{r}(-1)^{k}\left(\begin{array}{l}
r \\
k
\end{array}\right)\left(D_{1}^{r-k} D_{2}^{k} f\right)\left(D_{1}^{k} D_{2}^{r-k} \varphi\right) .
$$

It is convenient to omit the numerical factor and write as in [21, p. 57]

$$
\tau_{r}(f, \varphi)=\sum_{k=0}^{r}(-1)^{k}\left(\begin{array}{l}
r \\
k
\end{array}\right)\left(D_{1}^{r-k} D_{2}^{k} f\right)\left(D_{1}^{k} D_{2}^{r-k} \varphi\right) .
$$

Thus $\tau_{1}(f, \varphi)=J(f, \varphi)$ and $\tau_{2}(f, f)=2 H(f)$.

(3.10) Lemma. Suppose $f, \varphi$ are semi-invariant forms for $G$ with characters $\lambda, \mu$. Then $\tau_{r}(f, \varphi)$ is 0 or is a semi-invariant form with character $\delta^{r} \lambda \mu$.

Proof. Define the Cayley operator $\Omega: S \otimes S \rightarrow S \otimes S$ by $\Omega=D_{1} \otimes D_{2}$ $-D_{2} \otimes D_{1}$, and let $\pi: S \otimes S \rightarrow S$ be multiplication. If $g \in G$ then $\Omega(g f \otimes g \varphi)$ $=\delta(g) \Omega(f \otimes \varphi)$, and $\pi$ is a $G$-module homomorphism. The assertion follows since $\tau_{r}(f, \varphi)=\pi \Omega^{r}(f \otimes \varphi)$.

(3.11) Lemma. Let $f, \varphi \in S$ be forms of positive degrees $d, n$. Suppose $H(f) \neq 0$ and $\tau_{2}(f, \varphi)=0$. Let

$$
\mathbf{Q}=\left[\begin{array}{cc}
x_{1} & -D_{2} \varphi \\
x_{2} & D_{1} \varphi
\end{array}\right]
$$

and let $\psi=(n+d-2)^{-1} J(\varphi, f)$. Then $f, \psi$ are algebraically independent and $\mathbf{H}(f) \mathbf{Q}=(d-1) \mathbf{J}(f, \psi)$.

Proof. Note that the hypotheses imply $n \geq 1$ and $d \geq 2$ so $n+d-$ $2>0$. Direct computation using the Euler formula gives 


$$
\mathbf{H}(f) \mathbf{Q}=(d-1)\left[\begin{array}{ll}
D_{1} f & \psi_{1} \\
D_{2} f & \psi_{2}
\end{array}\right]
$$

where $\psi_{1}, \psi_{2}$ are defined by

(i) $(d-1) \psi_{1}=-\left(D_{1}^{2} f\right)\left(D_{2} \varphi\right)+\left(D_{1} D_{2} f\right)\left(D_{1} \varphi\right)$,

(ii) $(d-1) \psi_{2}=-\left(D_{1} D_{2} f\right)\left(D_{2} \varphi\right)+\left(D_{2}^{2} f\right)\left(D_{1} \varphi\right)$.

Since $H(f) \neq 0$ and $\operatorname{det} \mathbf{Q}=n \varphi \neq 0$, at least one of $\psi_{1}, \psi_{2}$ is not zero. Further computation gives $(d-1)\left(D_{1} \psi_{2}-D_{2} \psi_{1}\right)=\tau_{2}(f, \varphi)=0$. Thus $\psi_{1}, \psi_{2}$ are the partial derivatives of a form $\psi$ of degree $n+d-2$. Multiply (i) by $x_{1}$ and (ii) by $x_{2}$. The Euler formula gives

$(d-1)(n+d-2) \psi=-\left(x_{1} D_{1}^{2} f+x_{2} D_{1} D_{2} f\right)\left(D_{2} \varphi\right)+\left(x_{1} D_{1} D_{2} f+x_{2} D_{2}^{2} f\right)\left(D_{1} \varphi\right)$.

Since $D_{1} f, D_{2} f$ have degree $d-1$, application of the Euler formula to the right hand side proves $(n+d-2) \psi=J(\varphi, f)$. Thus $\mathbf{H}(f) \mathbf{Q}=(d-1) \mathbf{J}(f, \psi)$. Since $\operatorname{det} \mathbf{H}(f) \mathbf{Q}=n H(f) \varphi \neq 0$ it follows that $J(f, \psi) \neq 0$. This proves that $f, \psi$ are algebraically independent.

(3.12) Corollary. Let $f, \varphi \in S$ be forms of positive degree. Suppose $H(f) \neq 0$ and $\tau_{2}(f, \varphi)=0$. Then $J\left(D_{i} f, \varphi\right) \approx D_{i} J(f, \varphi)$ for $i=1,2$.

Proof. This follows by equating entries in the second column of the matrix equation in (3.11).

(3.13) Corollary. Let $f, \varphi \in S$ be forms of positive degree. Suppose $H(f) \neq 0$ and $\tau_{2}(f, \varphi)=0$. Then $J(f, J(\varphi, f)) \approx H(f) \varphi$.

Proof. This follows from (3.11) by taking determinants.

(3.14) Remark. The assertion in (3.13) is true without the hypothesis $H(f) \neq 0$. It follows from a known identity [6, p. 78] for the Jacobian of a Jacobian: if $f, \varphi, \psi$ are forms of degrees $d, n, p>1$ then

$$
J(J(f, \varphi), \psi) \approx \frac{d-n}{(d+n-2)}(f, \varphi)^{2} \psi+(f, \psi)^{2} \varphi-(\varphi, \psi)^{2} f
$$

where $(f, \varphi)=(f, \varphi)^{1}$ and $(f, \varphi)^{2}$ denote the transvectants defined in (3.9). Take $\psi=f$. The assertion follows since $(f, \varphi) \approx J(f, \varphi),(f, f)^{2} \approx H(f)$, and $(f, \varphi)^{2}=0$ by assumption.

(3.15) Theorem. Let $G \subset G L(V)$ be a Shephard group. Let $\eta_{1}=\theta_{E}$ be the Euler derivation and let $f_{1}$ be an invariant form of minimal positive degree. Define $\eta_{2} \in \operatorname{Der}_{S}$ and $f_{2} \in S$ by 


$$
\begin{gathered}
\eta_{2}=\left(-D_{2} Q\right) D_{1}+\left(D_{1} Q\right) D_{2} \\
d_{2} f_{2}=J\left(Q, f_{1}\right) .
\end{gathered}
$$

Then (i) $\eta_{1}, \eta_{2}$ are basic derivations, (ii) $f_{1}, f_{2}$ are basic invariants, and (iii) $\operatorname{Hess}\left(f_{1}\right) \eta_{i}=m_{1} d f_{i}$ for $i=1,2$.

Proof. Direct calculation shows that $\eta_{2} \in \operatorname{Der}_{S}^{G}$. Since $Q\left(\eta_{1}, \eta_{2}\right)=n Q$ it follows from (2.28) that $\eta_{1}, \eta_{2}$ are basic derivations. By (2.27) $Q$ is a semi-invariant with character $\delta^{-1}$. Since $f_{1}$ is invariant it follows from (3.10) that $\tau_{2}\left(f_{1}, Q\right)$ is either 0 or a semi-invariant form of degree $\left(d_{1}-2\right)$ $+(n-2)$ with character $\delta$. If $\tau_{2}\left(f_{1}, Q\right) \neq 0$ it follows from (2.26. iii) that $d_{1}+n-4 \geq m$ which contradicts Theorem (3.5). Thus

$$
\tau_{2}\left(f_{1}, Q\right)=0 \text {. }
$$

Since $H\left(f_{1}\right) \neq 0$ by (3.2), and $\tau_{2}\left(f_{1}, Q\right)=0$ it follows from (3.11) that $f_{1}$ and $f_{2}$ are algebraically independent. In particular $f_{2} \neq 0$. It follows from (3.5) that $\operatorname{deg} f_{2}=\operatorname{deg} J\left(Q, f_{1}\right)=(n-1)+\left(d_{1}-1\right)=d_{2}$. Since $Q$ is a semiinvariant with character $\delta^{-1}$ and $f_{1}$ is an invariant, it follows from (3.10) that $J\left(Q, f_{1}\right)$ is a semi-invariant with character $\delta \delta^{-1}=1$, so that $f_{2}$ is an invariant. Thus $f_{1}, f_{2}$ are algebraically independent invariant forms of degrees $d_{1}, d_{2}$ and hence are basic invariants. This proves (ii). Finally, since $n+d_{1}-2=d_{2}$ the matrix equation in (3.11) implies $\mathbf{H}\left(f_{1}\right) \mathbf{Q}\left(\eta_{1}, \eta_{2}\right)$ $=m_{1} \mathbf{J}\left(f_{1}, f_{2}\right)$. This proves (iii).

To complete the proof of (1.3) in case $\operatorname{dim} V=2$ let $f_{1}, f_{2}$ be the basic invariants constructed in (3.15). Since $d f_{i} \in i m \operatorname{Hess}\left(f_{1}\right)$ for $i=1,2$ and $\Omega_{S}^{G}=R d f_{1}+R d f_{2}$ it follows that Hess $\left(f_{1}\right)$ is surjective and hence by (3.2) it is an isomorphism.

We close this section with some observations on the connection between our work and Klein's polyhedral invariants. Let $G$ be the group $3[q] 3$ in Coxeter's notation [4, p. 94]. It is known from Coxeter's work that $3[q] 3$ is finite for $q=3,4,5$. Since $p_{i}=3$ for $i=1,2$ we have $\delta\left(s_{i}\right)^{3}$ $=\delta\left(s_{i}^{3}\right)=1$ so $\delta^{3}=1$ and thus $\delta^{4}=\delta$. Let $f=f_{1}$ be a $G$-invariant form of minimal positive degree. It follows from (3.10) that the fourth transvectant $(f, f)^{4}$ is 0 or it is a semi-invariant form with character $\delta$. Since $\operatorname{deg}(f, f)^{4}=2 d_{1}-8<d_{1}+d_{2}-2=m$, it follows from (2.26. iii) that $(f, f)^{4}$ $=0$. The binary forms $f$ of degree $n \geq 4$ which satisfy $(f, f)^{4}=0$ were characterized by Wedekind [25, pps. 42-51]. He showed that eithər $f=$ 
$\alpha^{n-1} \beta$ where $\alpha$ and $\beta$ are linear forms, allowing the possibility, $\alpha=\beta$, or that $n=4,6,12$ and $f$ lies in the $G L(V)$-orbit of the vertex form of one of the regular polyhedra with triangular faces: tetrahedron, octahedron, icosahedron. Since an irreducible group cannot have an invariant $\alpha^{n-1} \beta$ if $n \geq 3$, we may assume that $f \approx \varphi_{V}$ is a vertex form. There is something mysterious here because Wedekind's argument is a formal computation with the coefficients of a form $f$ which satisfies $(f, f)^{4}=0$. This leads him to a Diophantine equation which produces the integers 4, 6, 12 . One does not learn from his argument why the condition $(f, f)^{4}=0$ leads to a polyhedral group. The references given by Klein [9, pps. 62-63] have not helped us on this point.

Since $\delta^{3}=1$ we have $e_{H}=3$ for all $H \in \mathscr{A}$ and thus from (2.24) and (2.30) we get $H(f) \approx J / Q=\prod \alpha_{H}^{e_{H}-2}=\prod \alpha_{H}=Q$. Thus from Klein's formula (1.8) we have $Q \approx H\left(\varphi_{V}\right) \approx \varphi_{F}$. It follows from (3.17) that $f_{2} \approx J\left(f_{1}, Q\right) \approx$ $J\left(\varphi_{V}, \varphi_{F}\right) \approx \varphi_{E}$ where the last equality is Klein's (1.7). Thus for the groups $3[q] 3$ with $q=3,4,5$ we have

$$
f_{1} \approx \varphi_{V}, \quad Q \approx \varphi_{F}, \quad f_{2} \approx \varphi_{E} .
$$

Formula (1.10) follows from (3.12) with $f=\varphi_{V}$ and $\varphi=\varphi_{F}$. From (3.19) and (2.26) we have $J\left(\varphi_{V}, \varphi_{E}\right) \approx J\left(f_{1}, f_{2}\right) \approx J=\prod \alpha_{H}^{e_{H}^{-1}}=\prod \alpha_{H}^{2}=Q^{2} \approx \varphi_{F}^{2}$. This proves (1.8). It follows from (3.17) and (3.19) that $J\left(\varphi_{F}, \varphi_{E}\right) \approx J\left(Q, f_{2}\right)$ $\approx J\left(Q, J\left(Q, f_{1}\right)\right)$. Since $Q$ is not a power of a linear form, it follows from (3.1) that $H(Q) \neq 0$. Since $\tau_{2}(f, Q)=0$ we may replace $f$ by $Q$ and $\varphi$ by $f_{1}$ in (3.13) to conclude that $J\left(Q, J\left(Q, f_{1}\right)\right) \approx H(Q) f_{1} \approx H(Q) \varphi_{V}$. Thus the proof of (1.9) is reduced to showing that $H(Q) \approx f_{1}^{q-2}$. Since $Q$ is a semiinvariant with character $\delta^{-1}$ and $H(Q) \neq 0$ it follows from (3.10) that $H(Q)$ $\approx \tau_{2}(Q, Q)$ is a semi-invariant with character $\delta^{2} \delta^{-2}=1$. Now (3.5) shows that $H(Q)$ is an invariant form of degree $2 n-4<d_{2}$, and hence $H(Q)$ $\approx f_{1}^{\nu}$ where $\nu=(2 n-4) / d_{1}$. It follows from (3.5) and (3.8) that $2(n-2)=$ $2\left(d_{2}-d_{1}\right)=(q-2) d_{1}$. This proves (1.9). The vanishing of $(f, Q)^{2}$ proved in (3.18) is equivalent, for the groups $3[q] 3$, to the vanishing of $(f, f)^{4}$. This follows from the fact, remarked above, that $H(f) \approx Q$ and a formal identity [6, p. 52, Ex. 6]

$$
(H(f), f)^{2}=\frac{n-3}{2(2 n-5)}(f, f)^{4} f
$$

valid for all binary forms $f$ of degree $n$. 


\section{§4. The case $\operatorname{dim} V \geq 3$.}

The argument in Section 3 shows that in case $l=2$ Theorem (1.3) and hence Theorem (1.5) is a consequence of four facts: (i) $H(f) \neq 0$, (ii) $l\left(d_{1}-2\right)=m-n$, (iii) there is an explicit basis for $\operatorname{Der}_{s}^{(i}$ in terms of $Q$ and (iv) $\tau_{2}(f, Q)=0$. Both (i) and (ii) may be stated for arbitrary $l$ while (iii) and (iv) are special to $l=2$. In this section we verify (i) and (ii) for Shephard groups with $l \geq 3$ and prove that (i) and (ii) imply Theorem (1.5). We could have used this method for $l=2$ but it does not yield the explicit bases in (3.15) or the formulas (1.8)-(1.10) for Klein's polyhedral invariants.

Let $f \in S$ be any form. Hesse [7,8] claimed that if $H(f)=0$ then there exist linear forms $y_{1}, \cdots, y_{l-1} \in V^{*}$ such that $f \in C\left[y_{1}, \cdots, y_{l-1}\right]$. Sylvester [23], when told about this theorem, wrote that "an hour's quiet reflection in bed... sufficed to disclose to me the true principle of the solution". Hesse's assertion is easy to prove if $l=2$; see (3.1). It is much harder to prove if $l=3,4$. This was done by Gordan and Noether [5] who also showed that it is false if $l \geq 5$. See Noether's Note 30 in the appendix to Hesse's collected works [12], and the introduction to [5], for more history.

Suppose now that $G \subset G L(V)$ is any irreducible group. If $f$ is a $G$ invariant form of positive degree, then the map $v \rightarrow \operatorname{Hess}(f) D_{v}=d\left(D_{v} f\right)$ is a nonzero $G$-module homomorphism from $V$ into $S$. Since $G$ is an irreducible group this map is a monomorphism. Thus the partial derivatives $D_{1} f, \cdots, D_{l} f$ are linearly independent over $C$. It follows that there do not exist linear forms $y_{1}, \cdots, y_{l-1}$ with $f \in C\left[y_{1}, \cdots, y_{l-1}\right]$. Thus if Hesse's claim were correct we could conclude that $H(f) \neq 0$. As it stands, we know from Gordan and Noether that if $G \subset G L(V)$ is any irreducible group and $\operatorname{dim} V=2,3,4$ then $H(f) \neq 0$ for every $G$-invariant form of positive degree. We do not know if an irreducible group in dimension $\geq 5$ can have an invariant form $f$ of minimal positive degree with $H(f)$ $=0$.

(4.1) Lemma. Suppose $\operatorname{dim} V \geq 3$. If $G \subset G L(V)$ is a Shephard group and $f_{1}$ is a G-invariant form of minimal positive degree $d_{1}$ then (i) $H\left(f_{1}\right) \neq 0$ and (ii) $l\left(d_{1}-2\right)=m-n$.

Proof. Suppose first that $G$ is a Coxeter group. Since $G$ is irreducible it has a unique invariant quadratic form $f_{1}$ which is nondegenerate. Thus $H\left(f_{1}\right) \neq 0$. This proves (i). Assertion (ii) holds since $d_{1}=2$ and $m=n$. 
Suppose $G$ is not a Coxeter group. Then (ii) follows from the numbers listed in Table 1. See Appendix. Assertion (i) is clear if $G=G(p, 1, l)$ where $f_{1}=\sum x_{i}^{p}$. For the remaining groups $l=3,4$ so the assertion follows from the theorem of Gordan and Noether [5]. It is easy to check that $H\left(f_{1}\right) \neq 0$ directly using the invariants listed in Table 1 . In fact $H\left(C_{6}\right) \approx \widetilde{夭}_{12}$ and Maschke [11, p. 339] remarks that $H\left(F_{12}\right) \approx F_{40}$.

We use Lemma (4.1) and the results in Section 2 to prove Theorem (1.7). If $a_{1}, \cdots, a_{\imath}$ are the columns of a matrix $\mathbf{A}$ we write $\mathbf{A}=\left[a_{1}|\cdots| a_{\imath}\right]$.

(4.2) Lemma. Let $\mathbf{A}=\left[a_{1}|\cdots| a_{l}\right]$ and $\mathbf{B}=\left[b_{1}|\cdots| b_{l}\right]$ be $l \times l$ matrices with coefficients in a field. If $\mathbf{B}$ is invertible then

$$
\left(\mathbf{B}^{-1} \mathbf{A}\right)_{i j}=(\operatorname{det} \mathbf{B})^{-1} \operatorname{det}\left[b_{1}|\cdots| b_{i-1}\left|a_{j}\right| b_{i+1}|\cdots| b_{l}\right] .
$$

Proof. Let $c_{i j}=\left(\mathbf{B}^{-1} \mathbf{A}\right)_{i j}$ and let $c_{\jmath}=\left[c_{1 j}, c_{2 \jmath}, \cdots, c_{l j}\right]^{T}$. Then $\left[c_{1}|\cdots| c_{l}\right]$ $=\mathbf{B}^{-1} \mathbf{A}=\left[\mathbf{B}^{-1} a_{1}|\cdots| \mathbf{B}^{-1} a_{l}\right]$. Thus $\mathbf{B} c_{j}=a_{j}$. Fix $j$ and view this as a system of linear equations with given $a_{i j}$ and unknowns $c_{i j}$ for $i=1, \cdots, l$. The assertion of the lemma is Cramer's rule.

Table 1

\begin{tabular}{|c|c|c|c|c|c|c|c|}
\hline$S \& T$ & Coxeter & $m_{i}$ & $m$ & $n_{i}$ & $n$ & $f_{1}$ & $Q$ \\
\hline$G(p, 1, l)$ & $p[4] 2[3] \cdots 2$ & $\begin{array}{l}p-1,2 p-1 \\
\cdots, l p-1\end{array}$ & $\frac{p l(l+1)}{2}-l$ & $\begin{array}{l}1, p+1, \cdots \\
(l-1) p+1\end{array}$ & $\frac{p l(l-1)}{2}+l$ & $\sum x_{i}^{p}$ & $*$ \\
\hline 3 & {$[p]$} & $p-1$ & $p-1$ & 1 & 1 & $x^{p}$ & $x$ \\
\hline 4 & $3[3] 3$ & 3,5 & 8 & 1,3 & 4 & $\Phi$ & $\Psi$ \\
\hline 8 & $4[3] 4$ & 7,11 & 18 & 1,5 & 6 & $W$ & $t$ \\
\hline 16 & $5[3] 5$ & 19,29 & 48 & 1,11 & 12 & $H$ & $f$ \\
\hline 5 & $3[4] 3$ & 5,11 & 16 & 1,7 & 8 & $t$ & $W$ \\
\hline 10 & $4[4] 3$ & 11,23 & 34 & 1,13 & 14 & $\chi$ & $t W$ \\
\hline 18 & $5[4] 3$ & 29,59 & 88 & 1,31 & 32 & $T$ & $H f$ \\
\hline 20 & $3[5] 3$ & 11,29 & 40 & 1,19 & 20 & $f$ & $H$ \\
\hline 6 & $3[6] 2$ & 3,11 & 14 & 1,9 & 10 & $\Phi$ & $\Psi t$ \\
\hline 9 & $4[6] 2$ & 7,23 & 30 & 1,17 & 18 & $W$ & $t \chi$ \\
\hline 17 & $5[6] 2$ & 19,59 & 78 & 1,41 & 42 & $H$ & $f T$ \\
\hline 14 & $3[8] 2$ & 5,23 & 28 & 1,19 & 20 & $t$ & $W \chi$ \\
\hline 21 & $3[10] 2$ & 11,59 & 70 & 1,49 & 50 & $f$ & $H T$ \\
\hline 25 & $3[3] 3[3] 3$ & $5,8,11$ & 24 & $1,4,7$ & 12 & $C_{6}$ & $\mathfrak{夭}_{12}$ \\
\hline 26 & $3[3] 3[4] 2$ & $5,11,17$ & 33 & $1,7,13$ & 21 & $C_{6}$ & $C_{9} \Subset_{12}$ \\
\hline 32 & $3[3] 3[3] 3[3] 3$ & $11,17,23,29$ & 80 & $1,7,13,19$ & 40 & $F_{12}$ & $F_{40}$ \\
\hline
\end{tabular}


(4.3) Lemma. Let $G \subset G L(V)$ be a unitary reflection group. Let $f_{1}$, $\cdots, f_{l}$ be basic invariants and let $\mathbf{J}=\mathbf{J}\left(f_{1}, \cdots, f_{l}\right)$. Suppose $\mathbf{A} \in M_{l}(S)$ and $g \mathbf{A}=[g]^{T} \mathbf{A}$ for all $g \in G$. Then there exists a matrix $\mathbf{C} \in M_{l}(R)$ such that $\mathbf{A}=\mathbf{J C}$.

Proof. Write $\mathbf{A}=\left[a_{1}|\cdots| a_{l}\right]$ and $\mathbf{J}=\left[b_{1}|\cdots| b_{l}\right]$. Fix $g \in G$ and define $g a_{i}$ by $g \mathbf{A}=\left[g a_{1}|\cdots| g a_{l}\right]$. For simplicity of notation write $\mathbf{P}=[g]^{T}$. Since $g \mathbf{A}=\mathbf{P A}$ by assumption, and $g \mathbf{J}=\mathbf{P J}$ by (2.12), we have $g a_{i}=\mathbf{P} a_{i}$ and $g b_{i}=\mathbf{P} b_{i}$. For each $1 \leq i, j \leq l$ define $\mathbf{M}(i, j) \in M_{l}(S)$ by

$$
\mathbf{M}(i, j)=\left[b_{1}|\cdots| b_{i-1}\left|a_{j}\right| b_{i+1}|\cdots| b_{l}\right] \text {. }
$$

Then $g \mathbf{M}(i, j)=\mathbf{P M}(i, j)$. Thus $g(\operatorname{det} \mathbf{M}(i, j))=(\operatorname{det} \mathbf{P})(\operatorname{det} \mathbf{M}(i, j))=$ $(\operatorname{det} g)(\operatorname{det} \mathbf{M}(i, j))$. By (2.26. iii) we have $\operatorname{det} \mathbf{M}(i, j) \in R J$. By (2.26. ii) $\operatorname{det} \mathbf{J} \approx J \neq 0$ so $\mathbf{J}$ is invertible in $M_{l}(L)$ where $L$ is the quotient field of $S$. Let $\mathbf{C}=\mathbf{J}^{-1} \mathbf{A}$. It follows from (4.2) that $\mathbf{C}_{i j} \approx J^{-1} \operatorname{det} \mathbf{M}(i, j)$. Since $\operatorname{det} \mathbf{M}(i, j) \in R J$ we have $\mathbf{C}_{i j} \in R$.

(4.4) Remark. Let $G \subset G L(V)$ be a unitary reflection group. Let $\theta_{1}, \cdots, \theta_{l}$ be basic derivations and let $\mathbf{Q}=\mathbf{Q}\left(\theta_{1}, \cdots, \theta_{l}\right)$. If $\mathbf{A} \in M_{l}(S)$ and $g \mathbf{A}=\left[g^{-1}\right] \mathbf{A}$ for all $g \in G$ then there exists a matrix $\mathbf{C} \in M_{l}(R)$ such that $\mathbf{A}=\mathbf{Q C}$. The argument is similar to that in (4.3).

(4.5) Proposition. Let $G \subset G L(V)$ be a unitary reflection group. Let $f_{1}, \cdots, f_{l}$ be basic invariants and let $\theta_{1}, \cdots, \theta_{l}$ be basic derivations. If $f \in R$ then there exists $\mathbf{C} \in M_{l}(R)$ such that

$$
\mathbf{H}(f) \mathbf{Q}\left(\theta_{1}, \cdots, \theta_{l}\right)=\mathbf{J}\left(f_{1}, \cdots, f_{l}\right) \mathbf{C} .
$$

If $f$ is homogeneous then the nonzero entries of $\mathbf{C}$ are homogeneous.

Proof. Write $\mathbf{J}=\mathbf{J}\left(f_{1}, \cdots, f_{l}\right)$ and $\mathbf{Q}=\mathbf{Q}\left(\theta_{1}, \cdots, \theta_{l}\right)$. It follows from (2.11) and (2.13) that $g(\mathbf{H}(f) \mathbf{Q})=[g]^{T} \mathbf{H}(f) \mathbf{Q}$ so we may apply (4.3) to find a matrix $\mathbf{C}$ in $M_{l}(R)$ which satisfies $\mathbf{H}(f) \mathbf{Q}=\mathbf{J C}$. The nonzero entries of a given column of $\mathbf{J}$ or $\mathbf{Q}$ are homogeneous polynomials all of which have the same degree. If $f$ is homogeneous then all nonzero entries of $\mathbf{H}(f)$ have the same degree. Thus the nonzero entries of $\mathbf{C}$ are homogeneous.

(4.6) Remark. Note that (2.29. ii) follows from (4.5) by taking determinants.

Now we may complete the proof of Theorem (1.3). By (4.1) we have 
$H\left(f_{1}\right) \neq 0$ so Hess $\left(f_{1}\right)$ is injective. Choose a set of basic derivations $\varphi_{1}$, $\cdots, \varphi_{l}$, To prove that Hess $(f)$ is surjective we modify $\varphi_{1}, \cdots, \varphi_{l}$ to produce a new set $\theta_{1}, \cdots, \theta_{l}$ of basic derivations such that Hess $\left(f_{1}\right) \theta_{i}=m_{1} d f_{i}$. To denote the dependence of $\mathbf{Q}$ on the basic derivations we write $\mathbf{Q}(\varphi)$ or $\mathbf{Q}(\theta)$ in this argument. By (4.5) there exists $\mathbf{C} \in M_{l}(R)$ such that $\mathbf{H}(f) \mathbf{Q}(\varphi)$ $=$ JC. Let $C=\operatorname{det} \mathbf{C}$. Then $H(f) Q(\varphi)=J C$. Comparing degrees we get $l\left(d_{1}-2\right)+n=m+\operatorname{deg} C$. It follows from (4.1) that $C$ is not zero. Thus $\mathbf{C}$ is invertible in $M_{l}(R)$. Define $\theta_{1}, \cdots, \theta_{l} \in \operatorname{Der}_{S}^{G}$ by $m_{1} \varphi_{j}=\sum \mathbf{C}_{k j} \theta_{k}$. Then $m_{1} \mathbf{Q}(\varphi)=\mathbf{Q}(\theta) \mathbf{C}$ and hence $\mathbf{H}(f) \mathbf{Q}(\theta)=m_{1} \mathbf{J}$. This proves that Hess $(f) \theta_{i}=$ $m_{1} d f_{i}$. Since $f$ is homogeneous and the $d f_{i}$ are homogeneous, the $\theta_{i}$ may be replaced by suitable homogeneous components and thus may be assumed homogeneous. Since $Q(\theta) \approx Q(\varphi)$ it follows that $\theta_{1}, \cdots, \theta_{l}$ are basic derivations.

\section{$\S 5$. Consequences of the Main Theorem}

In this section we derive some consequences of Theorems (1.3) and (1.5). Write $\mathbf{J}=\mathbf{J}\left(f_{1}, \cdots, f_{l}\right)$ and $\mathbf{Q}=\mathbf{Q}\left(\theta_{1}, \cdots, \theta_{l}\right)$.

(5.1) Corollary. Let $G \subset G L(V)$ be a Shephard group. Let $f_{1}, \cdots, f_{l}$ be basic invariants and let $\theta_{1}, \cdots, \theta_{l}$ be basic derivations such that $\mathbf{H}\left(f_{1}\right) \mathbf{Q}$ $=m_{1} \mathbf{J}$. Then $\theta_{\imath}\left(f_{\jmath}\right)=\theta_{j}\left(f_{i}\right)$.

Proof. Since $\mathbf{H}\left(f_{1}\right)$ is symmetric so is $m_{1} \mathbf{J}^{T} \mathbf{Q}=\mathbf{Q}^{T} \mathbf{H}\left(f_{1}\right) \mathbf{Q}$. The assertion follows since $\theta_{j} f_{i}=\sum \theta_{j}\left(x_{k}\right) D_{k} f_{i}=\left(\mathbf{J}^{T} \mathbf{Q}\right)_{i j}$.

(5.2) Remark. If follows from (2.9) that the natural $S$-bilinear pairing $\Omega_{S} \times \operatorname{Der}_{S} \rightarrow S$ restricts to an $R$-bilinear pairing $\Psi: \Omega_{S}^{G} \times \operatorname{Der}_{S}^{G} \rightarrow R$ for which $\Psi(d f, \theta)=\theta(f)$. The matrix for $\Psi$ with respect to the bases $f_{1}, \cdots, f_{l}$ and $\theta_{1}, \cdots, \theta_{l}$ is $\mathbf{J}^{T} \mathbf{Q}$. Each $f \in S$ defines a symmetric $S$-bilinear form $\operatorname{Der}_{S}$ $\times \operatorname{Der}_{S} \rightarrow S$ given by $(\theta, \eta) \rightarrow\langle\operatorname{Hess}(f) \theta, \eta\rangle$. If $f \in R$ this form restricts to a symmetric $R$-bilinear form $\Phi_{f}: \operatorname{Der}_{S}^{G} \times \operatorname{Der}_{S}^{G} \rightarrow R$ given by $\Phi_{f}(\theta, \eta)=$ $\Psi(\operatorname{Hess}(f) \theta, \eta)$. The matrix for $\Phi_{f}$ with respect to the basis $\theta_{1}, \cdots, \theta_{l}$ is $\mathbf{Q}^{T} \mathbf{H}(f) \mathbf{Q}$. The two pairings $\Psi$ and $\Phi_{f}$ are defined for all unitary reflection groups. If $G$ is a Shephard group and $f=f_{1}$ then $\mathbf{H}\left(f_{1}\right) \mathbf{Q}=m_{1} \mathbf{J}$ shows that their matrices $m_{1} \mathbf{J}^{T} \mathbf{Q}=\mathbf{Q}^{T} \mathbf{H}\left(f_{1}\right) \mathbf{Q}$ are the same.

(5.3) Corollary. Let $G \subset G L(V)$ be a Shephard group. Then $m_{i}-n_{i}$ $=d_{1}-2$ is a non-negative integer independent of $i$.

Proof. Choose $f_{1}, \cdots, f_{l}$ and $\theta_{1}, \cdots, \theta_{l}$ as in (1.4). Since deg $d f_{3}=$ 
$m_{i}+1, \operatorname{deg} \theta_{i}=n_{i}-1$ and $\operatorname{deg} \operatorname{Hess}\left(f_{1}\right)=d_{1}$ we have $n_{i}-1+d_{1}=m_{i}+1$.

(5.4) Corollary. Let $G \subset G L(V)$ be a Shephard group. An invariant form of minimal positive degree is unique up to a constant multiple.

Proof. We have $m_{1}+n_{2}=m_{2}+n_{1}$ from (5.3) and $1=n_{1}<n_{2}$ from (2.23). Thus $d_{1}<d_{2}$.

(5.5) Corollary. Let $G \subset G L(V)$ be a Shephard group and let $f \in R$ be any invariant. Then $\left(D_{1} f, \cdots, D_{l} f\right) \subseteq\left(D_{1} f_{1}, \cdots, D_{l} f_{1}\right)$.

Proof. Since $R=C\left[f_{1}, \cdots, f_{l}\right]$ it suffices to show this if $f=f_{k}$ is a basic invariant. Since $\mathbf{H}\left(f_{1}\right) \mathbf{Q}=m_{1} \mathbf{J}$ we have

$$
\sum_{j}\left(D_{i} D_{j} f_{1}\right)\left(\theta_{k} x_{j}\right)=m_{1} D_{i} f_{k} .
$$

Multiply by $x_{i}$, sum over $i$ and use the Euler formula. This gives

$$
\sum_{j}\left(D_{j} f_{1}\right)\left(\theta_{k} x_{j}\right)=d_{k} f_{k}
$$

Apply $D_{i}$ to (5.7):

$$
\sum_{j}\left(D_{i} D_{j} f_{1}\right)\left(\theta_{k} x_{j}\right)+\sum_{j}\left(D_{j} f_{1}\right)\left(D_{i} \theta_{k} x_{j}\right)=d_{k}\left(D_{i} f_{k}\right) .
$$

By (5.6) the first sum is $m_{1} D_{i} f_{k}$ so we have

$$
\sum_{j}\left(D_{j} f_{1}\right)\left(D_{i} \theta_{k} x_{j}\right)=\left(d_{k}-m_{1}\right) D_{i} f_{k} .
$$

(5.8) Corollary. Let $G \subset G L(V)$ be a Shephard group and let $f_{1}, \cdots$, $f_{l}$ be basic invariants. Then (i) $\left(f_{1}, \cdots, f_{l}\right) \subset\left(D_{1} f_{1}, \cdots, D_{l} f_{1}\right)$ and (ii) a form $f_{1}$ of minimal positive degree is nondegenerate.

Proof. The first assertion follows from (5.5); in fact (5.7) expresses $f_{k}$ as an $S$-linear combination of the $D_{j} f_{1}$. Since $S$ is integral over $R$ the origin is the only common zero of $f_{1}, \cdots, f_{l}$. It follows from (i) that the origin is the only common zero of $D_{1} f_{1}, \cdots, D_{l} f_{1}$.

(5.9) Remark. There are irreducible unitary reflection groups for which the assertions in (5.3), (5.4), (5.5), and (5.8) are false. For example, in the groups numbered 7,11, 19 by Shephard and Todd, all these assertions are false. Note also that there are finite irreducible Coxeter groups which are not the automorphism groups of regular polytopes and hence not Shephard groups. 
(5.10) Theorem. Let $G \subset G L(V)$ be a finite irreducible unitary reflection group. The following statements are equivalent:

(i) $G$ is a Shephard group or a Coxeter group,

(ii) Hess $\left(f_{1}\right): \operatorname{Der}_{S}^{G} \rightarrow \Omega_{S}^{G}$ is an isomorphism,

(iii) $m_{i}-n_{i}=d_{1}-2$ for $1 \leq i \leq l$,

(iv) $m-n=l\left(d_{1}-2\right)$,

(v) $H\left(f_{1}\right) \approx \prod_{H \in \mathscr{W}} \alpha_{H}^{e_{H}-2}$.

Proof. If $G$ is a Shephard group then (i) $\Rightarrow$ (ii) is Theorem (1.3), the main result of this paper. If $G$ is a Coxeter group then $f_{1}$ is a nondegenerate quadratic form and (i) $\Rightarrow$ (ii) follows as we remarked in the Introduction. The argument used to prove Corollary (5.3) shows that (ii) $\Rightarrow$ (iii). The assertion (iii) $\Rightarrow$ (iv) follows from (2.25) and (iv) $\Rightarrow$ (v) follows from (2.30). Clearly (v) $\Rightarrow$ (iv) by comparing degrees. The assertion (iv) $\Rightarrow$ (i) follows from the information in [13, Table 2] and the list of Shephard groups in Table 1 of this paper. We expect a proof of (iv) $\Rightarrow$ (i), which does not use the classification, to be difficult.

\section{Appendix}

In Table 1 we list those Shephard groups which are not Coxeter groups. Column 1 labels the groups as in Table VII of [19]; Shephard and Todd indicate which groups are symmetry groups of regular complex polytopes. Column 2 gives Coxeter's symbol for the groups as in [4]. The numbers in the columns headed $m_{i}, m, n_{i}, n$ are given in [13, Table 2]. The column headed $f_{1}$ gives an invariant polynomial of degree $d_{1}=m_{1}+1$. The column headed $Q$ gives a polynomial $\prod_{H \in \mathscr{A}} \alpha_{H}$. The last two columns depend on choice of coordinates in $V$. If $l=2$ we use the polynomials $\Phi, \Psi, t, W, \chi, f, H, T$ of Klein [9]. In case $l=3,4$ we use the polynomials $C_{6}, C_{9}, \Im_{12}, F_{12}, F_{40}$ of Maschke [11]. The invariants $f_{1}$ are listed by Shephard and Todd, who use different letters for these polynomials.

\section{REFERENCES}

[1] N. Bourbaki, Groupes et algèbres de Lie, Chapitres 4, 5 et 6. Hermann, Paris, 1968.

[2] H. S. M. Coxeter, The Symmetry Groups of the Regular Complex Polygons, Arch. Math., 13 (1962), 86-97.

[ 3 ] H. S. M. Coxeter, Finite groups generated by unitary reflections, Abh. Math. Sem. Univ. Hamburg, 31 (1967), 127-135.

[4] —-, Regular Complex Polytopes, Cambridge Univ. Press, 1974. 
[5] P. Gordan and M. Noether, Ueber die algebraischen Formen, deren Hesse'sche Determinante identisch verschwindet, Math. Ann., 10 (1876), 547-568.

[6] J. H. Grace and A. Young, The Algebra of Invariants, Cambridge Univ. Press, 1903.

[ 7 ] L. O. Hesse, Ueber die Bedingung, unter welcher eine homogene ganze Function von $\mathrm{n}$ unabhängigen Variabeln durch lineäre Substitutionen von $\mathrm{n}$ anderen unabhängigen Variabeln auf eine homogene Function sich zurückführen lässt, die eine Variable weniger enthält, J. reine angew. Math., 42 (1852), 117-124, reprinted in Gesammelte Werke, Chelsea, 1972.

[ 8 ] _- Zur Theorie der ganzen homogenen Functionen, J. reine angew. Math., 56 (1859), 481-496, reprinted in Gesammelte Werke, Chelsea, 1972.

[ 9 ] F. Klein, Lectures on the Icosahedron, Kegan Paul, London 1913, reprinted Dover 1956.

[10] D. W. Koster, Complex Reflection Groups, Thesis, Univ. of Wisconsin, Madison, 1975.

[11] H. Maschke, Aufstellung des vollen Formensystems einer quaternären Gruppe von 51840 linearen Substitutionen, Math. Ann., 33 (1888), 317-344.

[12] M. Noether, Anmerkungen zu den Abhandlungen L. O. Hesse, Note 30, p. 704 in L. O. Hesse, Gesammelte Werke, Chelsea, 1972.

[13] P. Orlik and L. Solomon, Unitary reflection groups and cohomology, Invent. math., 59 (1980), 77-94.

[14] - Discriminants in the invariant theory of reflection groups, Nagoya Math. J., 109 (1988), 23-45.

[15] K. Saito, On a linear structure of a quotient variety by a finite reflection group, RIMS Report 288, Kyoto, 1979.

[16] - Theory of logarithmic differential forms and logarithmic vector fields, J. Fac. Sci. Univ. Tokyo, Sect. IA, 27 (1980), 265-291.

[17] G. C. Shephard, Regular Complex Polytopes, Proc. London Math. Soc., (3) 2 (1952), 82-97.

[18] — Unitary Groups Generated by Reflections, Canad. J. Math., 5 (1953), 364383.

[19] G. C. Shephard and J. A. Todd, Finite Unitary Reflection Groups, Canad. J. Math., 6 (1954), 274-304.

[20] L. Solomon, Invariants of Euclidean reflection groups, Trans. Amer. Math. Soc., 113 (1964), 274-286.

[21] T. A. Springer, Invariant Theory, Lecture Notes in Math. vol. 585, Springer Verlag, 1977.

[22] — - Regular elements of finite reflection groups, Invent. math., 25 (1974), 159198.

[23] J. J. Sylvester, On the conditions necessary and sufficient to be satisfied in order that a function of any number of variables may be linearly equivalent to a function of any less number of variables, Phil. Mag., 5 (1853), 119-126, reprinted in Collected Works I, Chelsea 1973.

[24] H. Terao, Free Arrangements of Hyperplanes and Unitary Reflection Groups, Proc. Japan. Acad., 56, Ser. A (1980), 389-392.

[25] L. Wedekind, Studien im Binären Werthgebiet, Habilitationsschrift Polytechnikum zu Carlsruhe, Carlsruhe, 1876. 